\title{
Potential errors when fitting experience curves by means of spreadsheet software
}

\author{
W.G.J.H.M. van Sark*, E.A. Alsema \\ Department of Science, Technology and Society, Copernicus Institute, Utrecht University, Heidelberglaan 2, 3584 CS Utrecht, The Netherlands
}

\section{A R T I C L E I N F O}

\section{Article history:}

Received 24 March 2010

Accepted 29 June 2010

Available online 31 July 2010

Keywords:

Experience curve

Progress ratio

Data transformation

\begin{abstract}
A B S T R A C T
Progress ratios (PRs) are widely used in forecasting development of many technologies; they are derived from historical data represented in experience curves. Fitting the double logarithmic graphs is easily done with spreadsheet software like Microsoft Excel, by adding a trend line to the graph. However, it is unknown to many that these data are transformed to linear data before a fit is performed. This leads to erroneous results or a transformation bias in the $P R$, as we demonstrate using the experience curve for photovoltaic technology: logarithmic transformation leads to overestimates of progress ratios and underestimates of goodness of fit. Therefore, other graphing and analysis software is recommended.
\end{abstract}

(c) 2010 Elsevier Ltd. All rights reserved.

\section{Introduction}

Technology development can conveniently be represented by the so-called experience or learning curve that in its mostly used form depicts the costs of the technology of a functional unit representative of the technology as a function of cumulative amount of produced units (Boston Consulting Group, 1972; Dutton and Thomas, 1984; McDonald and Schrattenholzer, 2001; Wene, 2000; Junginger, et al., 2010). For example, in case of many renewable technologies, the costs are given in US\$, Euro $(€)$, or Yen ( $¥)$, per Watt-peak (Wp), and are plotted as a function of cumulative produced capacity in MWp. These empirical curves illustrate that technical and economic performance of a technology increases substantially as producers and consumers gain experience with this technology. When these curves are plotted on a double-logarithmic scale, they are straight lines, which can be easily extrapolated in order to forecast future technology development. The slope of these lines usually are found using curve fitting. In this paper we will show that the choice of the right method for curve fitting is very important and that the use of Microsoft Excel software to make the fit can lead to erroneous results.

\section{Experience curve methodology}

In many studies experience curves have been constructed on the basis of historical data that span several decades (Dutton and Thomas, 1984; McDonald and Schrattenholzer, 2001). Such a

\footnotetext{
* Corersponding author. Tel.: +3130253 7611; fax: +31302537601

E-mail address: w.g.j.h.m.vansark@uu.nl (W. van Sark).
}

curve can be described as

$c_{x}=a x^{m}$

in which $c_{x}$ is the costs required to produce the $x$ th unit of production, $x$ the cumulative production up to and including the $x$ th unit of production, $a$ the costs required to produce the first unit, and $m$ the measure of the rate of costs reduction as cumulative production increases. Eq. (1) can conveniently be rewritten in logarithmic form

$\log c_{x}=\log a+m \log x$

The constant parameter $m$ also is denoted learning or experience parameter, and is used to calculate the progress ratio $P R$ for cumulative doubling of production

$P R=\frac{c_{x_{2}}}{c_{x_{1}}}=\frac{a x_{2}^{m}}{a x_{1}^{m}}=2^{m} \quad\left(\right.$ for $\left.x_{2}=2 x_{1}\right)$

The learning rate $L R$ is then defined as one minus the progress ratio: $L R=1-P R$. Progress ratios have been found to vary between 0.5 and 1.0 for the semiconductor industry (Albright, 2002), manufacturing firms (Dutton and Thomas, 1984), and energy technologies (McDonald and Schrattenholzer, 2001; Junginger, et al., 2010).

As experience curve data are empirical in nature with inherent uncertainties, the $P R$ is likely to vary to some extent when key parameters are changed such as the assumptions about initial capacity installed, the associated start-off costs, the method of aggregating annual data, correcting for inflation and varying exchange rates and changing the learning system boundaries (Junginger, et al., 2005). It is important to realize that small changes in $P R$ lead to large variations in energy model outcomes 
in which experience curves are employed to model endogenous learning (Neij, 1999; Sagar and Van der Zwaan, 2006; Van der Zwaan and Seebregts, 2004). For example, Van der Zwaan and Rabl (Van der Zwaan and Rabl, 2003) report that a variation of the progress ratio for photovoltaics (PV) technology has an enormous influence on the cost of reaching break-even, where the breakeven unit cost is taken as $1 \$ / \mathrm{Wp}$. For a progress ratio of 0.80 the cost to reach break-even is reported to be 211 billion US\$, which is reduced to one-third for a progress ratio of 0.75 . We therefore have recommended (Van Sark, 2008) to attach an uncertainty to the $P R$ value, e.g., $0.80 \pm 0.05$, thus indicating the range of possible values scenario developers should use in sensitivity studies.

\section{Results and discussion}

\subsection{Fitting}

Fitting experience curves is easily and usually done with Microsoft Excel spreadsheet software, by adding the trend line denoted as "power" to a double logarithmic graph. As an example, we have plotted and fitted the recently updated experience curve for photovoltaic technology (Van Sark, et al., 2008) using Excel, see Fig. 1. The PV experience curve was constructed for the period 1976-2006 using data from Strategies Unlimited.(Strategies Unlimited, 2003), Swanson (Swanson, 2006), and Hirschman et al. (Hirschman, et al., 2007). We find $a=43.243, m=-0.3127$, and $P R=0.805$, with the coefficient of determination $R^{2}=0.983$, which corresponds well with the reported $P R$ of 0.81 by Swanson (Swanson, 2006) who used the period 1976-2005.

However, when using another data analysis and graphing software such as Origin (Ledvij, 2003), we find different results. In Fig. 2 we have plotted the same data as in Fig. 1 and used the nonlinear curve fitting tool "allometric1" to find $a=45.3 \pm 0.7$, $m=-0.333 \pm 0.008$, and $P R=0.794 \pm 0.003$, with $R^{2}=0.992$. As Origin also gives the errors in the parameters $a$ and $m$, we are able to determine the error in $P R$, as reported in (Van Sark, 2008). The value of PR determined using Excel $(P R=0.805)$ clearly is larger than the one determined by Origin, and is not even within the error margin of 0.003 .

The question now arises: why do different software programs yield results that are significantly different, while the fitting functions are identical, i.e., $y=a x^{m}$, and the quality of the fits is high as both values of $R^{2}$ indicate?

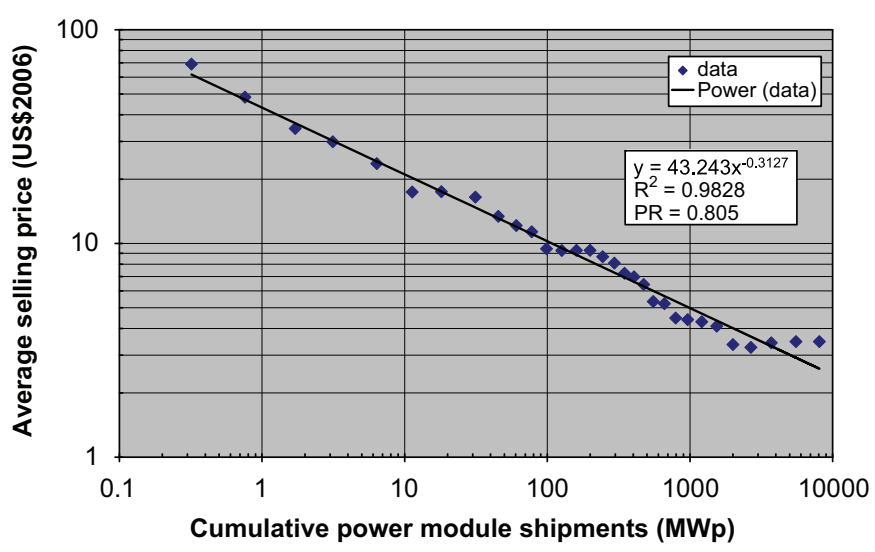

Fig. 1. PV experience curve made with Excel, showing average module price in 2006 US\$ as a function of cumulative power module shipments in MWp for the period 1976-2006. Data from Strategies Unlimited (Strategies Unlimited, 2003) are combined with data from Swanson (Swanson, 2006) and Hirschman et al. (Hirschman et al., 2007).

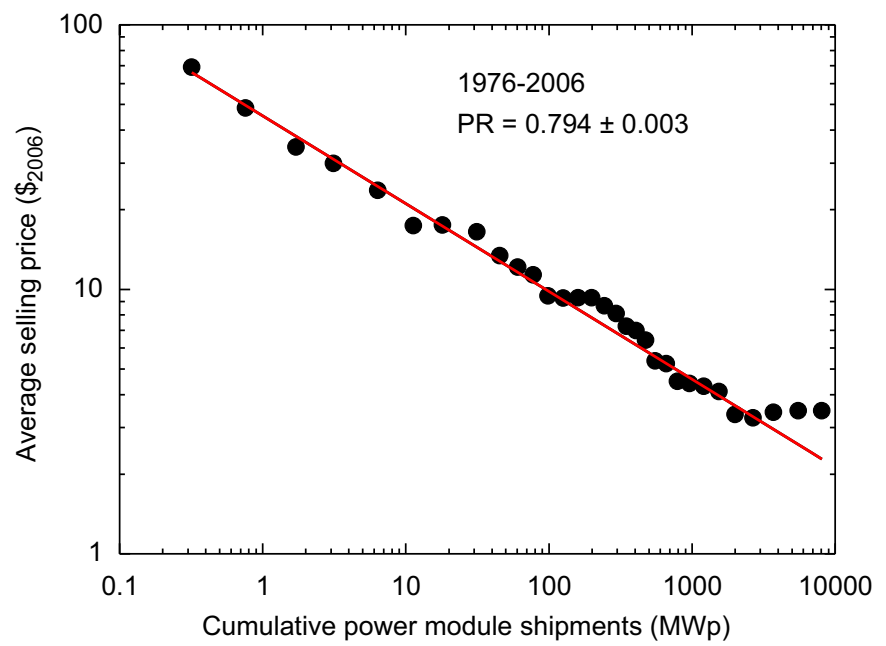

Fig. 2. PV experience curve made with Origin, using the same data as in Fig. 1.

Using Origin we found that if the original data are first transformed to linear data, i.e., by taking the ${ }^{10} \log$ of both $c_{x}$ and $x$, and subsequently fitted with a linear function, i.e., Eq. (2), the resulting $P R$ is identical to the one found using Excel: log $a=1.636 \pm 0.019, \quad m=-0.313 \pm 0.008$, and $P R=0.805 \pm 0.004$, with $R^{2}=0.983$. We therefore conclude that in Excel the data are first linearized before the "power" trend line is added. As this is not known to the user, he is unaware of the possibly erroneous result. The online documentation via the Help function of Excel does not inform the user how the trend line is calculated. Mehta (2010) reports that the function LINEST is used after linearization of the data.

\subsection{Explanation}

As the differences in found $P R$ values are statistically significant, we need to find the correct value. We will discuss this by considering fitting principles. The experience curve as shown in Eq. (1) can be generalized as

$y=f(x ; a, m)$

in which $y$ is the dependent variable, $x$ the independent variable, $f($ ) the function describing the dependency between $y$ and $x$, and $a$ and $m$ the parameters used in the function $f()$. The so-called chisquare $\left(\chi_{r}^{2}\right)$ minimization is often performed to find the best fit by using the Levenberg-Marquardt method (Levenberg, 1944; Marquardt, 1963): it minimizes the sum of the squares of the vertical deviations between the experimental curve and a (non)linear theoretical curve of choice. Finding the best fit to the function is based on the assumption that the vertical deviations from the fitted line (or fitting residues defined as $r=y-f(x ; a, m)$ ) are distributed according to a normal distribution (Bevington, 1969; Ledvij, 2003), i.e., Gaussian shaped. If we plot these deviations for direct fitting, i.e., using Origin, we clearly observe a Gaussian shape and the data are distributed with only small skewness (Abramowitz and Stegun, 1972); fitting yields the line as shown in Fig. 3a. If we first take the logarithm followed by linear fitting, the deviations still are Gaussian-shaped; however fitting to a Gaussian distribution yields a much worse fit than obtained with direct fitting, and the data are clearly negatively skewed (Fig. 3b). We therefore tentatively conclude that transformation of the data also transforms the Gaussian shape normal distribution, and fitting no longer is valid. 
a

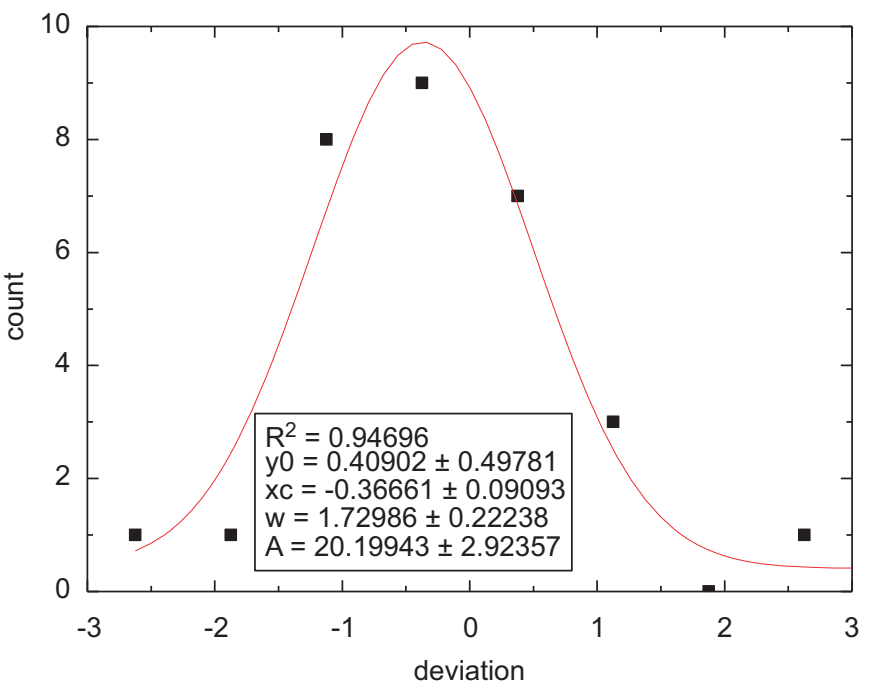

b

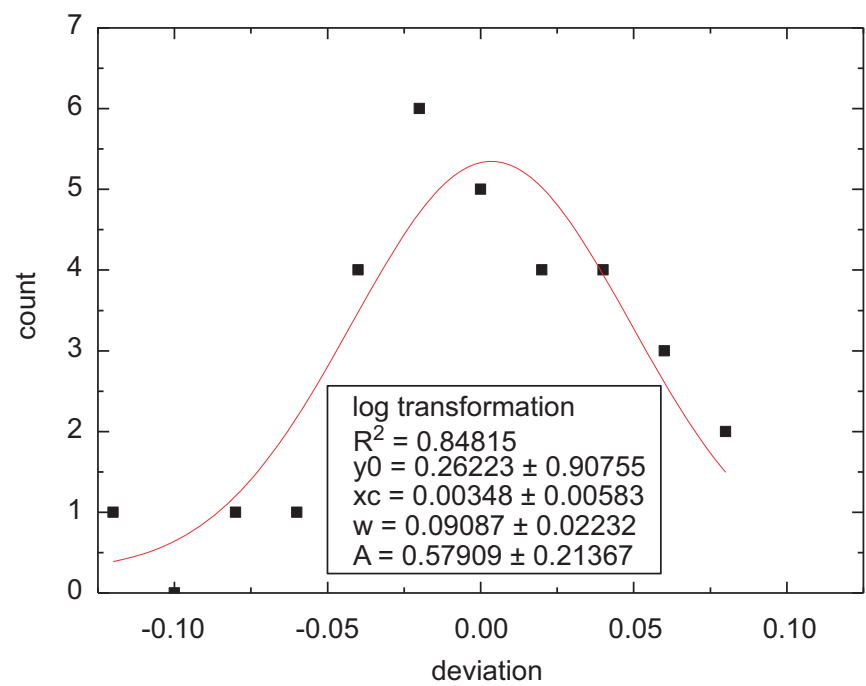

Fig. 3. Distribution of fitting residues for (a) direct fitting using a power function, and (b) linear fitting after logarithmic data transformation.

\subsection{Fitting generated data}

As in the example above the values of $R^{2}$ are high, and the difference in $P R$ is small, albeit significant, we have generated other data sets, that by means of direct fitting yield the same value of $P R$, but have a lower value of $R^{2}$, To this end, we simply multiplied the fitting residues by 2 and 4 , and added them to the fitted data points. The results of both fitting methods are shown in Table 1. Clearly, and as expected, larger fitting residues lead to smaller $R^{2}$ values and larger errors in $P R$. More troublesome, however, is the fact that with increasing fitting residues (and smaller $R^{2}$ ), the differences between the two fitting methods become progressively larger. This even more proves that data transformation introduces a bias in the determination of the $P R$, and leads to larger values of $P R$. In addition, the goodness of the fit becomes worse, as reflected in the lower $R^{2}$ value.

\subsection{Other studies}

In many studies, experience curves are presented with $R^{2}$ values lower than the ones presented here for the case of
Table 1

Comparison of the two procedures for fitting the original data, and data with 2 and 4 times the original fitting residue.

\begin{tabular}{lllllll}
\hline & Direct & & \multicolumn{5}{c}{ Log transformation } \\
\hline Fitting residue & 1 & 2 & 4 & 1 & 2 & 4 \\
$P R$ & 0.794 & 0.794 & 0.794 & 0.805 & 0.813 & 0.829 \\
$\sigma_{P R}$ & 0.003 & 0.007 & 0.013 & 0.004 & 0.008 & 0.015 \\
$R^{2}$ & 0.992 & 0.970 & 0.883 & 0.983 & 0.937 & 0.781 \\
\hline
\end{tabular}

photovoltaic technology. As it is usually not stated which fitting software is used, the PRs presented in these studies should be used with care. As we have shown for the PV experience curve, even if $R^{2}$ values as high as 0.98 are found, the associated $P R$ value will be estimated too high if it is obtained by a procedure that transforms the data before the actual fit is made.

As an additional example to substantiate the above statement we have analysed the data presented on wind energy development (Junginger et al., 2005) for wind farms in Spain and the UK for the periods 1990-2001 (Spain) and 1992-2001 (UK). Reported values for $P R$ are $P R=0.805 \pm 0.010\left(R^{2}=0.978\right)$ for the UK and $P R=0.851 \pm 0.016\left(R^{2}=0.887\right)$ for Spain, where the errors were determined using the method described by Van Sark (2008). Note that $P R$ values were determined by using Sigmaplot. Analysis using Excel yields $P R=0.811\left(R^{2}=0.970\right)$ for the UK and $P R=0.862$ $\left(R^{2}=0.899\right)$ for Spain. In both cases fitting results from Excel yield higher values for $P R$; however, for the UK case, the difference $(0.006)$ is within the error margin, while for the Spain case (difference is 0.011 ) this is not so.

From the examples given in this paper, a logarithmic transformation bias error of about 0.01 in $P R$ may be deduced, which may seem small, but which already can have serious consequences in scenario studies. A larger database of studies is needed to determine the effect of logarithmic transformation in the case of data for which $R^{2}$ is smaller than for the cases shown in this paper.

\section{Conclusion}

In conclusion, we have shown that determination of the progress ratio from experience curves with historical price data should be performed with care. Small variations in the progress ratio will give large differences when the experience curve is extrapolated, for example in scenario studies.

We have found that standard spreadsheet software, such as Microsoft Excel, performs a double-log fit by first linearizing the data and subsequently determining the best fit. We have shown that such a data transformation followed by a linear fit yields results that are principally erroneous; the assumptions underlying the fitting procedure are no longer valid after transformation. Using standard spreadsheet software for a double-log fit may thus result in a serious misestimation of the fit parameters, even in case of relatively "good fits" with high $R^{2}$ values. Thus if one wants to correctly determine a progress ratio from experience curve data the proper power function should be used.

As the user is unaware of the fact that data transformation followed by linear fitting is used in Microsoft Excel (it cannot be found in the online manual) when adding a power trend line, we recommend using other analysis and graphing software, such as Origin, Sigmaplot, or Mathematica. Alternatively, macros for Excel for non-linear curve fitting are described by Billo (2007) could be used. 


\section{Acknowledgements}

The authors would like to acknowledge stimulating discussions with Hendriek Boshuizen (National Institute for Public Health and the Environment, RIVM, the Netherlands) and Martin Junginger (Utrecht University).

\section{References}

Abramowitz, M., Stegun, I.A., 1972. Handbook of Mathematical Functions. Dover Publications, New York, USA.

Albright, R.E., 2002. What can past technology tell us about the future. Technol Forecast. Soc. Change 69, 443-464.

Bevington, P.R., 1969. Data Reduction and Error Analysis for the Physical Sciences. McGraw-Hill, New York, NY, USA.

Billo, E.J., 2007. Excel for Scientist and Engineers. Wiley, Hoboken, NJ, USA

Boston Consulting Group, 1972. Perspectives on Experience. BCG, Boston.

Dutton, J.M., Thomas, A., 1984. Treating progress functions as a managerial opportunity. Acad. Man. Rev. 9, 235-247.

Hirschman, W.P., Hering, G., Schmela, M., 2007. Gigawatts-the measure of things to come. Market survey on global solar cell and module production in 2006. Photon Int. March, 136-166.

Junginger, M., Faaij, A., Turkenburg, W.C., 2005. Global experience curves for wind farms. Energy Pol. 33, 133-150.

Junginger, M., Faaij, A., Van Sark, W. (Eds.), 2010. Technological Learning in the Energy Sector, Lessons for Policy, Industry and Science. Edward Elgar, Cheltenham, UK.
Ledvij, M., 2003. Curve fitting made easy. Ind. Phys. 9, 24-27.

Levenberg, K., 1944. A method for the solution of certain nonlinear problems in least squares. Q. Appl. Math. 2, 164-168.

Marquardt, D.W., 1963. An algorithm for least-squares estimation of nonlinear parameters. J. Soc. Ind. Appl. Math. 11, 431-441.

McDonald, A., Schrattenholzer, L., 2001. Learning rates for energy technologies. Energy Pol. 29, 255-261.

Mehta, T., 2010. Trendline coefficients and regression analysis, 〈http://www. tushar-mehta.com/publish_train/data_analysis/16.htm $\rangle$ (accessed 28 June 2010).

Neij, L., 1999. Cost dynamics of wind power. Energy 24, 375-389.

Sagar, A.D., Van der Zwaan, B., 2006. Technological innovation in the energy sector: R\&D, deployment, and learning-by-doing. Energy Pol. 34, 2601-2608.

Strategies Unlimited, 2003. Photovoltaic Five-Year Market Forecast 2002-2007, Mountain View, CA, USA.

Swanson, R.M., 2006. A vision for crystalline silicon photovoltaics. Prog. Photovolt: Res. Appl. 14, 443-453.

Van der Zwaan, B., Rabl, A., 2003. Prospects for PV: a learning curve analysis. Sol. Energy 74, 19-31.

Van der Zwaan, B.C.C., Seebregts, A., 2004. Endogenous learning in climate-energyeconomic models - an inventory of key uncertainties. Int. J. Energy Technol. Policy 2, 130-141.

Van Sark, W.G.J.H.M., 2008. Introducing errors in progress ratios determined from experience curves. Technol. Forecast. Soc. Change 75, 405-415.

Van Sark, W.G.J.H.M., Alsema, E.A., Junginger, H.M., De Moor, H.H.C., Schaeffer, G.J., 2008. Accuracy of progress ratios determined from experience curves: the case of photovoltaic technology development. Prog. Photovolt: Res. Appl. 16, 441-453.

Wene, C.-O., 2000. Experience Curves for Energy Technology Policy. OECD/IEA, Paris. 